\title{
Neuropsychological detection and characterization of preclinical Alzheimer's disease
}

\author{
D.M. Jacobs, PhD; M. Sano, PhD; G. Dooneief, MD; K. Marder, MD; K.L. Bell, MD; and Y. Stern, PhD
}

\begin{abstract}
Article abstract-We attempted to characterize the changes in cognition associated with the earliest, or preclinical, stages of Alzheimer's disease (AD) by administering a comprehensive neuropsychological test battery to a group of initially nondemented older adults participating in a prospective epidemiologic study of dementia. Using Cox regression analyses, we examined the associations between baseline neuropsychological test scores and subsequent development of AD. Results confirmed preliminary findings that baseline scores on the Boston Naming Test, Immediate Recall on the Selective Reminding Test, and the Similarities subtest of the Wechsler Adult Intelligence Scale--Revised were significantly and independently associated with later diagnosis of $\mathrm{AD}$. Analyses controlled for the effects of age, education, sex, and language of test administration. These results lend support to the notion of a preclinical phase of $\mathrm{AD}$ and indicate that this very early stage of $\mathrm{AD}$ is characterized by poor word-finding ability, abstract reasoning, and memory.
\end{abstract}

NEUROLOGY 1995;45:957-962

Early detection of Alzheimer's disease (AD) is important both for clinical care of patients and for improving our understanding of this disorder. Because neuropsychological tests can detect very subtle cognitive impairment, they may be sensitive to early changes in cognition that might occur well before frank dementia becomes apparent. Therefore, neuropsychological tests may be useful for identifying individuals in the very earliest, or preclinical, stages of $\mathrm{AD}$.

Previous studies examining the neuropsychological characteristics of preclinical dementia generally have focused on the assessment of memory. Fuld et al, ${ }^{1}$ Masur et $a l,{ }^{2}$ and Tuokko et $\mathrm{l}^{3}$ prospectively followed cohorts of initially nondemented elderly people and compared baseline memory testing of subjects who later became demented with the baseline testing of subjects whose cognitive functioning remained unchanged. Results from these studies indicate that performance on the Fuld Object Memory Evaluation, ${ }^{1}$ the Buschke Selective Reminding Test (SRT), ${ }^{2}$ and a cued recall task ${ }^{3}$ differentiates subjects who later become demented from those who remain nondemented.

Since memory impairment is a defining feature of dementia, tests of memory should be useful for identifying patients who are in the incipient stages of a dementing disorder. Masur et $\mathrm{al}^{4}$ recently reported that in addition to two memory measures, baseline scores on the Digit Symbol subtest of the Wechsler Adult Intelligence Scale-Revised (WAIS$R)^{5}$ and a verbal fluency measure independently contributed to the prediction of later dementia in their cohort of initially healthy elderly people from the Bronx Aging Study. Similarly, preliminary results from our cohort of initially nondemented elderly subjects in the North Manhattan Aging Project indicate that tests of verbal abstract reasoning and word-finding are significant predictors of later dementia, independent of performance on memory testing. ${ }^{6}$ Our finding was based upon a matched case-control comparison of 26 initially nondemented subjects who developed dementia by 1-year follow-up and 26 subjects who remained nondemented. We evaluated the predictive value of each test in a comprehensive neuropsychological test battery to identify at baseline those subjects who would subsequently be diagnosed as having dementia. The measures with the highest positive predictive value $(\mathrm{PV}+)$ (ie, percentage of subjects identified as at risk who actually became demented) were a 15-item version of the Boston Naming Test (PV+ $=90 \%$ ), Immediate Recall on the Selective Reminding Test (PV+ $=88 \%$ ), and WAIS-R Similarities $(\mathrm{PV}+=79 \%)$. In contrast to previous reports, Delayed Recall on the Selective Reminding Test was not a good predictor of later dementia $(\mathrm{PV}+=50 \%)$. We conducted the current study to confirm these

From the Gertrude H. Sergievsky Center (Drs. Jacobs, Sano, Dooneief, Marder, Bell, and Stern), and Departments of Neurology (Drs. Jacobs, Sano, Dooneief, Marder, Bell, and Stern) and Psychiatry (Dr. Stern), Columbia University College of Physicians and Surgeons, New York, NY.

Supported by Federal grants AG07232, AG08702, and RR00645 and the Charles S. Robertson Gift for Alzheimer's Disease.

Presented in part at the annual meeting of the American Academy of Neurology, Washington, DC, May 1994.

Received July 11, 1994. Accepted in final form October 25, 1994

Address correspondence and reprint requests to Dr. Diane M. Jacobs, Gertrude H. Sergievsky Center, 630 West 168 th Street, New York, NY 10032. 
preliminary findings in a larger sample of prospectively followed older adults from our communitybased cohort.

Methods. Subjects. Data were obtained from subjects participating in the North Manhattan Aging Project, a community-based, epidemiologic study of dementia in northern Manhattan, NY. All subjects included in the analyses presented here had completed at least one annual follow-up evaluation. Because we were interested in defining the neuropsychological characteristics of preclinical $\mathrm{AD}$, subjects diagnosed as demented at baseline were excluded from these analyses. In addition, subjects with any of the following diagnoses at baseline were excluded: "questionable"7 or borderline dementia, stroke, Parkinson's disease, major depression, or a cognitive impairment of known etiology (eg, head trauma).

Procedures. Subjects were evaluated annually, and received the same standardized assessment at each study visit. A physician conducted a standardized physical and neurologic examination and recorded each subject's medical history. All available ancillary information, including medical records and imaging studies, was used in the evaluation. In addition, a brief (approximately 1 hour) neuropsychological test battery was administered. The neuropsychological battery was designed to assess a broad range of cognitive functions, and consisted primarily of subtests and short forms of widely used neuropsychological measures. Evaluations were conducted in either English or Spanish, based on the subject's primary language and opinion of which language would yield better performance. To assure comparability of evaluations in English and Spanish, all interview questions, test instructions, and stimuli were translated into Spanish and then translated back to ensure accuracy. Specific cognitive functions assessed and neuropsychological tests administered include the following:

Word list learning and memory. The SRT ${ }^{8}$ was administered. Subjects were given six trials to learn a list of 12 unrelated words. After each recall attempt, a selective reminding procedure was used wherein subjects were reminded only of those words that had not been successfully recalled. To assess long-term retention of the word list, 15-minute delayed free recall was assessed, followed by a multiple-choice recognition task. The predictive value of summed total recall across the six acquisition trials, total delayed recall, and total correct recognition was assessed.

Nonverbal memory. A multiple-choice version of the Benton Visual Retention Test (BVRT) ${ }^{9}$ was used to assess nonverbal memory. Subjects viewed a geometric design for 10 seconds. It was then removed from view, and the subject was asked to recognize the design in a fourchoice multiple-choice array. Stimuli corresponded to Form D of the original BVRT.

Orientation. The 10 orientation items from the MiniMental State Examination ${ }^{10}$ were used to assess orientation to time and place.

Verbal reasoning. The Similarities subtest of the WAIS-R, ${ }^{5}$ which requires subjects to identify relevant similarities or superordinate categories for paired items, was administered. The age-corrected scaled score was used in analyses.

Nonverbal reasoning. The Identities and Oddities subtest of the Mattis Dementia Rating Scale ${ }^{11}$ was used to assess nonverbal reasoning. In this test, subjects identify which two of three visually presented stimuli are the same. After all eight triads are completed, the same items are administered again, and the subject is required to identify the one item that is different.

Naming. A 15-item version of the Boston Naming Test, ${ }^{12}$ a test of visual confrontation naming, was used to assess word-finding ability.

Verbal fluency. Letter and category fluency were assessed. For each trial, subjects were given 1 minute to generate as many words as possible. English-speaking subjects generated words beginning with the letters $\mathrm{C}, \mathrm{F}$, and L, while Spanish-speaking subjects generated words beginning with the letters $\mathrm{P}, \mathrm{S}$, and V. Different letters were used for Spanish- and English-speaking subjects to control for word-frequency differences across the two languages: the letters C, F, L in English and P, S, V in Spanish are comparable in terms of number of words beginning with these letters. All subjects generated exemplars in the categories animals, foods, and clothing. Average fluency across the three trials for each type of task were used for analysis.

Auditory comprehension. The first six items of the Complex Ideational Material subtest of the Boston Diagnostic Aphasia Examination ${ }^{13}$ were used to assess comprehension of spoken language.

Repetition. Subjects were asked to repeat the high-frequency phrases from the Boston Diagnostic Aphasia Examination Repetition of Phrases subtest. ${ }^{13}$

Visuoconstructional skills. Subjects copied five designs from the Rosen Drawing Test ${ }^{14}$ ranging in difficulty from simple geometric shapes to overlapping, parallel, and three-dimensional figures.

Visuoperceptual skills. Subjects matched a target design to the same design presented simultaneously in a four-choice multiple-choice array containing the target along with three distractors. Target stimuli corresponded to Form $\mathrm{C}$ of the original BVRT. ${ }^{9}$

Medical, historical, and neuropsychological data were reviewed at a diagnostic conference of physicians and neuropsychologists and a consensus diagnosis of the presence or absence of dementia was made. The diagnosis of dementia was based on the criteria of the DSM-III$\mathrm{R}^{15}$ and required evidence of cognitive deficit on the neuropsychological battery as well as evidence of impairment in social or occupational function. When dementia was diagnosed, all available data were examined to determine the etiology. NINCDS-ADRDA criteria ${ }^{16}$ were used for the diagnosis of probable and possible $\mathrm{AD}$.

Data analysis. We used Cox proportional hazards models ${ }^{17}$ to assess the relative risk (RR) of incident dementia associated with baseline scores on the Boston Naming Test, WAIS-R Similarities, and Immediate Recall on the SRT. Duration of follow-up was used as the timing variable for Cox analyses. For incident dementia cases with more than one follow-up visit, we used duration from baseline to the first follow-up visit at which dementia was diagnosed; for subjects who remained nondemented, we used duration from baseline to the last available follow-up visit. Age, education, sex, and language of test administration (English versus Spanish) were included in the model as covariates. All variates and covariates were entered into the model simultaneously; thus, the RR associated with each variable was independent of all other variates and covariates.

Two supplementary Cox analyses also were performed. In one analysis, additional neuropsychological measures were entered in a stepwise fashion to determine whether any of the other variables in our test battery provided an independent contribution to the predictor model above and beyond our three a priori selected 
measures (ie, Boston Naming Test, WAIS-R Similarities, and SRT Immediate Recall). Second, since previous reports have suggested that delayed recall on the SRT is a good predictor of subsequent dementia in initially nondemented elderly people, ${ }^{2}$ we performed a Cox regression using Delayed Recall rather than Immediate Recall on the SRT as the measure of memory function.

Results. At least one follow-up evaluation was available for 449 subjects who had no baseline diagnosis of dementia or other disorder likely to affect cognition. Six of these cases were excluded from analyses because of missing data. The remaining 443 subjects were included in the Cox analyses. The mean age of the subject sample was $73.32( \pm 7.05)$ years, and the average level of education was $10.60( \pm 4.60)$ years. The group was $73 \%$ women and $38 \%$ Spanish-speaking. The overall average duration of follow-up was $2.05( \pm 0.81)$ years.

Forty-one of the 443 initially nondemented subjects had diagnosed $A D$ at follow-up. Demographic characteristics of subjects who did and did not become demented are presented in table 1. Patients with incident dementia were older and less educated than subjects who remained nondemented. There were no significant differences between the two groups in distribution of sex or language.

Thirty-one of the 41 patients with incident dementia had a diagnosis of probable $\mathrm{AD}$; the remaining 10 were diagnosed as having possible $\mathrm{AD}$ with other concomitant illnesses that were thought possibly to have contributed to the change in mental status but did not completely account for it. Concomitant illnesses included cerebrovascular accidents (CVAs), hypothyroidism, and systemic illnesses. There were no incident cases of vascular dementia. This is likely due, in part, to the fact that we excluded from these analyses subjects with a history of stroke at baseline. The clinical course in those subjects diagnosed as having possible $\mathrm{AD}$ and concomitant CVA was consistent with possible $\mathrm{AD}$, including insidious onset of symptoms and gradual decline. In some cases, the strokes were clinically "silent," but evident on CT.

When baseline test scores were treated as continuous variables, a lower score on the Boston Naming Test, WAIS-R Similarities, or SRT Immediate Recall was significantly and independently associated with increased risk for subsequent $\mathrm{AD}$ diagnosis, even after controlling for age, educational attainment, sex, and language of test administration (see table 2). Age and sex also provided a significant contribution to the Cox model. When the Cox regression was repeated with only the 31 subjects diagnosed as having probable $\mathrm{AD}$ included as incident cases, the Boston Naming Test $(\beta=0.7$; $95 \% \mathrm{CI}=0.6$ to 0.8 ), WAIS-R Similarities $(\beta=0.7$; $95 \% \mathrm{CI}=0.6$ to 0.9$)$, and SRT Immediate Recall $(\beta$ $=0.9 ; 95 \% \mathrm{CI}=0.8$ to 0.9 ) remained strong and independent predictors of subsequent dementia.

In order to aid in the interpretation of RR values, we also dichotomized baseline test perfor-
Table 1. Characteristics of subjects who did and did not become demented

\begin{tabular}{|lcc|}
\hline & $\begin{array}{c}\text { Incident } \\
\text { dementia } \\
(\mathbf{N}=\mathbf{4 1})\end{array}$ & $\begin{array}{c}\text { No incident } \\
\text { dementia } \\
(\mathbf{N}=\mathbf{4 0 2})\end{array}$ \\
& $79.41(8.60)$ & $72.70(6.58)$ \\
Mean (SD) age & $7.46(4.14)$ & $10.95(4.55)$ \\
Mean (SD) education & & \\
(yr) & 78 & 73 \\
Sex (\% female) & 46 & 37 \\
Language (\% Spanish) & 32 & 26 \\
Duration of follow-up* & 54 & 45 \\
1 year & 10 & 54 \\
2 years & 5 & 5 \\
3 years & & \\
4 years & & \\
* Percentage of each group completing follow-up evaluations 1 to 4 \\
years after baseline. For incident dementia cases, duration from \\
baseline to the first follow-up visit at which dementia was diag- \\
nosed is reported. For subjects who remained nondemented, dura- \\
tion from baseline to the last available follow-up visit is reported. \\
\hline
\end{tabular}

Table 2. Risk ratios for incident Alzheimer's disease associated with baseline test scores

\begin{tabular}{|c|c|c|c|}
\hline \multicolumn{2}{|c|}{ Variable* } & $\begin{array}{l}\text { Adjusted } \\
\text { risk ratio }\end{array}$ & $\begin{array}{c}95 \% \text { Confidence } \\
\text { interval }\end{array}$ \\
\hline \multicolumn{2}{|l|}{ Age } & 1.1 & $1.0-1.1$ \\
\hline \multicolumn{2}{|c|}{ Education } & 0.9 & $0.9-1.0$ \\
\hline \multicolumn{2}{|c|}{ Sex (female) } & 2.5 & $1.1-5.9$ \\
\hline \multicolumn{2}{|c|}{ Language (Spanish) } & 0.6 & $0.3-1.3$ \\
\hline \multicolumn{2}{|c|}{ Boston Naming Test } & 0.8 & $0.7-0.9$ \\
\hline \multicolumn{2}{|c|}{ WAIS-R Similarities } & 0.8 & $0.6-0.9$ \\
\hline \multicolumn{2}{|c|}{ SRT Immediate Recall } & 0.9 & $0.9-1.0$ \\
\hline \multicolumn{4}{|c|}{$\begin{array}{l}\text { * Variables were continuous, with the exception of sex and } \\
\text { language. Adjusted risk ratios for continuous variables } \\
\text { represent relative risk of incident dementia per unit } \\
\text { change (per point difference) independent of the other } \\
\text { variables in the model. } \\
\text { WAIS-R Wechsler Adult Intelligence Scale-Revised. } \\
\text { SRT Buschke Selective Reminding Test. }\end{array}$} \\
\hline
\end{tabular}

mance into impaired and unimpaired ranges, and examined the RR associated with a score in the impaired range. Impairment was defined as a score more than two SDs below the mean scores of a group of 322 cognitively normal older adults from the same community-based cohort. Scoring in the impaired range on the Boston Naming Test, WAIS$\mathrm{R}$ Similarities, or SRT Immediate Recall was significantly and independently associated with increased risk for subsequent $\mathrm{AD}$ diagnosis, again after controlling for age, education, sex, and language of test administration (see table 3). Further, the risk of subsequent $\mathrm{AD}$ associated with baseline scores in the impaired range on any two of these measures increased approximately five-fold and, if baseline scores on all three measures were in the impaired range, twelve-fold.

Supplementary analyses. Results of the Cox analyses with stepwise inclusion of additional neu- 
Table 3. Risk ratios for incident Alzheimer's disease associated with baseline test scores in the impaired range ( $2 \mathrm{SD}$ below the mean)

\begin{tabular}{|lcc|}
\hline Variable & $\begin{array}{c}\text { Adjusted } \\
\text { risk ratio }\end{array}$ & $\begin{array}{c}\text { 95\% Confidence } \\
\text { interval }\end{array}$ \\
Age & 1.1 & $1.1-1.2$ \\
Education & 0.9 & $0.8-1.0$ \\
Sex (female) & 1.7 & $0.7-3.9$ \\
Language (Spanish) & 0.7 & $0.3-1.4$ \\
Boston Naming Test & 2.4 & $1.2-4.7$ \\
WAIS-R Similarities & 2.5 & $1.2-5.1$ \\
SRT Immediate Recall & 2.1 & $1.0-4.2$ \\
WAIS-R Wechsler Adult Intelligence Scale-Revised. \\
SRT Buschke Selective Reminding Test. \\
\hline
\end{tabular}

ropsychological measures revealed that the variable with the greatest additional contribution to the predictor model (ie, after inclusion of the Boston Naming Test, WAIS-R Similarities, SRT. Immediate Recall, and the demographic covariates) was our measure of nonverbal memory, BVRT Recognition ( $R R=1.29 ; 95 \% \mathrm{CI}=1.09$ to 1.52$)$. After BVRT Recognition was included in the model, no other variable provided a significant independent contribution.

There was a high correlation between SRT Immediate and Delayed Recall ( $r=0.74 ; p<0.0001$ ), so when both variables were included in the model, neither made a significant independent contribution. Therefore, a separate Cox analysis was performed that included SRT Delayed Recall, rather than Immediate Recall, in the model with Boston Naming Test, WAIS-R Similarities, and the demographic covariates (ie, age, education, sex, and language). When treated as a continuous variable, poor baseline performance on SRT Delayed Recall was associated with a significantly increased risk of subsequent $\mathrm{AD}$ diagnosis $(\mathrm{RR}=1.4 ; 95 \% \mathrm{CI}=1.2$ to 1.7). When dichotomized, however, scoring in the impaired range at baseline on SRT Delayed Recall was not associated with increased risk of later $\mathrm{AD}$ $(\mathrm{RR}=2.4 ; 95 \% \mathrm{CI}=0.7$ to 8.6$)$.

Discussion. The results of this study confirm our preliminary findings and corroborate the recently published report of Masur et $\mathrm{al}^{4}$ indicating that the preclinical stages of $\mathrm{AD}$ are characterized by more than just memory impairment. We found that changes in abstract reasoning and confrontation naming ability occur well before clinical signs of dementia are evident. Evaluation of these cognitive abilities in the context of a comprehensive dementia evaluation may assist in detecting $\mathrm{AD}$ in its earliest stages.

The neuropsychological characteristics of preclinical $\mathrm{AD}$ described here are consistent with previous reports of psychometric test performance in subjects with very mild $\mathrm{AD} .{ }^{18-20}$ Welsh et al ${ }^{18}$ reported that delayed recall on a list-learning task was the best psychometric measure for differentiating early $\mathrm{AD}$ from normal aging, and the only nonmemory measure from the Consortium to Establish a Registry for Alzheimer's Disease (CERAD) neuropsychological battery to assist in this discrimination was an abbreviated version of the Boston Naming Test. Storandt and Hill ${ }^{19}$ and Morris et al ${ }^{20}$ also found poor confrontation naming in addition to impaired memory and psychomotor speed in very mildly demented $A D$ patients. Our results suggest that the impairments in memory, naming, and verbal reasoning, often prominent in the early stages of dementia, can be detected even before functional impairment is evident and dementia is diagnosed.

Our results lend further support to the notion of a preclinical phase of $\mathrm{AD}$. The neuropsychological measures detected subtle deficits of memory, abstraction, and word-finding abilities in nondemented subjects who only later met criteria for $A D$. Although we cannot be certain that all of the 41 incident dementia cases were not already clinically demented at baseline, that the subjects were demented and our procedures simply failed to detect it is unlikely. At baseline, no subject had difficulty in social or occupational functioning due to cognitive impairment. Each subject was examined by a behavioral neurologist who assessed mental status without prior knowledge of the subject's performance on neuropsychological testing. Neurologists diagnosed all subjects as nondemented at baseline, with four exceptions (two incident $A D$ cases and two subjects who remained nondemented). At the diagnostic consensus conference, where a team of neurologists and neuropsychologists reviewed subjects' medical and social histories and neuropsychological test scores, diagnoses for these four subjects were changed to nondemented. For completeness, the Cox models were re-run without these four subjects, and results essentially were unchanged; the three neuropsychological measures remained significant and independent predictors of subsequent dementia. Although the diagnosis of dementia was based in part on the neuropsychological tests, we never based diagnoses solely on these measures but considered subjects' day-to-day functioning, medical histories, examination findings, and test results in the diagnostic process. We never diagnosed dementia in the absence of a history of functional decline, regardless of neuropsychological test scores.

In addition to the independent neurologic and neuropsychological assessment of each subject, a potentially useful adjunct to our assessment procedures would be an informant-based rating of subject's functional status. Koss et $\mathrm{al}^{21}$ and Morris et $\mathrm{al}^{20}$ demonstrated that collateral sources, such as spouses or caregivers, generally appraise and report accurately cognitive and functional deficits in $\mathrm{AD}$ patients, even those with very mild or "questionable" dementia. Implementing informant-based assessments in our community-based cohort has been difficult, however, because many of the subjects live alone, and a reliable informant may not be available. 
The neuropsychological characteristics of preclinical $A D$ differ in magnitude and scope from those associated with age-associated memory impairment (AAMI). The diagnostic criteria for AAMI include complaints of memory loss and objective memory test performance that is at least $1 \mathrm{SD}$ below the normative mean for young adults, in the absence of dementia and presence of adequate intellectual function. ${ }^{22}$ Koivisto et al $^{23}$ recently reported a high prevalence rate of AAMI $(38.4 \%)$ in a randomly selected population of older adults from eastern Finland, and concluded that AAMI characterizes a phenomenon of normal aging rather than a distinct clinical entity. In contrast, our initially nondemented subjects who subsequently developed $\mathrm{AD}$ scored lower on neuropsychological tests not only relative to young adults, but also when compared with peers of comparable age. In addition, poor naming and verbal abstract reasoning independently were associated with preclinical AD. Performance on nonmemory tests of cognitive function are not addressed in the diagnostic criteria for AAMI.

The current study has several advantages compared with some of the previous prospective neuropsychological investigations of preclinical dementia. Fuld et al, ${ }^{1}$ Masur et $a{ }^{,}{ }^{2}$ and Tuokko et $\mathrm{al}^{3}$ restricted the assessment of cognition in their nondemented elderly subjects to measures of memory. In the current study, we, like Masur et al, ${ }^{4}$ used a comprehensive neuropsychological test battery that assessed a range of cognitive domains. By systematically assessing diverse cognitive abilities, we were able to delineate various intellectual skills that are affected early in the course of $\mathrm{AD}$. A potential limitation of our study, however, is that the tasks within our neuropsychological battery may not be of comparable difficulty. Therefore, additional abilities, for example constructional skills, may also be affected in preclinical $\mathrm{AD}$, but our instruments may not be sensitive enough to detect subtle changes. Nevertheless, our finding that naming and abstract reasoning are affected in preclinical $A D$, in addition to memory, corroborates and extends the results of Masur et $\mathrm{al}^{4}$ that cognitive abilities other than memory are impaired in nondemented older adults who subsequently develop clinically significant dementia.

A second advantage of the current study is our use of Cox modeling to assess the RR of incident dementia associated with baseline test scores. Previous investigations generally have relied upon univariate estimates of test sensitivity, specificity, and predictive value. The benefits of Cox analysis include the inclusion of time in the predictor model; thus, it is not necessary to limit follow-up to a specific interval. In addition, by including duration of longitudinal follow-up in the model, Cox analysis controls for differences across subjects in terms of the opportunity to reach the endpoint (ie, become demented). Cox analysis also provides the ability to assess the effects of one variate while simultane- ously controlling for the effects of other variates and covariates. Previous studies often have failed to address potential differences between incident cases and subjects who remain nondemented in terms of demographic variables that can affect neuropsychological test performance, such as age and level of education. Failure to control for these important confounders can lead to erroneous interpretation of the neuropsychological data.

As is common to many clinical studies of $A D$, our findings are limited by inability to validate the diagnosis of probable or possible $\mathrm{AD}$. Although 16 of the subjects included in the analyses presented here (four incident dementia cases and 12 subjects who remained nondemented) have died, none had a postmortem examination. Thus, we are unable to confirm the diagnosis of probable or possible $\mathrm{AD}$ in the incident cases.

\section{References}

1. Fuld PA, Masur DM, Blau AD, Crystal H, Aronson MK. Object memory evaluation for prospective detection of dementia in normal functioning elderly: predictive and normative data. J Clin Exp Neuropsychol 1990;12:520-528.

2. Masur DM, Fuld PA, Blau AD, Crystal H, Aronson MK. Predicting development of dementia in the elderly with the selective reminding test. J Clin Exp Neuropsychol 1990; 12:529-538.

3. Tuokko H, Vernon-Wilkinson R, Weir J, Beattie BL. Cued recall and early identification of dementia. J Clin Exp Neuropsychol 1991;13:871-879.

4. Masur DM, Sliwinski M, Lipton RB, Blau AD, Crystal HA. Neuropsychological prediction of dementia and absence of dementia in healthy elderly persons. Neurology 1994; 44:1427-1432.

5. Wechsler D. Wechsler Adult Intelligence Scale-Revised. New York: Psychological Corp, 1981.

6. Jacobs D, Sano M, Albert S, Stern Y. Neuropsychological characteristics of preclinical Alzheimer's disease [abstract]. Neurology 1994;44(suppl 2):A354.

7. Rubin EH, Morris JC, Grant EA, Vendegna T. Very mild senile dementia of the Alzheimer type. I. Clinical assessment. Arch Neurol 1989;46:379-382.

8. Buschke H, Fuld PA. Evaluating storage, retention, and retrieval in disordered memory and learning. Neurology 1974; 24:1019-1025.

9. Benton AL. The Visual Retention Test. New York: Psychological Corp, 1955.

10. Folstein MF, Folstein SE, McHugh PR. 'Mini-mental State': a practical method for grading the cognitive state of patients for the clinician. J Psychiatr Res 1975;12:189-198.

11. Mattis S. Mental status examination for organic mental syndrome in the elderly patient. In: Bellak L, Karasu TB, eds. Geriatric psychiatry. New York: Grune \& Stratton, 1976:77121.

12. Kaplan E, Goodglass H, Weintraub S. Boston Naming Test. Philadelphia: Lea \& Febiger, 1983.

13. Goodglass H, Kaplan E. The assessment of aphasia and related disorders. 2nd ed. Philadelphia: Lea \& Febiger, 1983.

14. Rosen W. The Rosen Drawing Test. Bronx, NY: Veterans Administration Medical Center, 1981.

15. American Psychiatric Association. Diagnostic and statistical manual of mental disorders. Rev 3rd ed. Washington, DC: American Psychiatric Press, 1987.

16. McKhann G, Drachman D, Folstein M, Katzman R, Price D, Stadlan E. Clinical diagnosis of Alzheimer's disease: report of the NINCDS-ADRDA Work Group under the auspices of the Department of Health and Human Services Task Force on Alzheimer's Disease. Neurology 1984;34:939-944. 
17. Cox DR. Regression models and life-tables. J R Stat Soc [B] 1972;34:187-220.

18. Welsh KA, Butters N, Hughes JP, Mohs RC, Heyman A. Detection and staging of dementia in Alzheimer's disease. Arch Neurol 1992;49:448-452.

19. Storandt M, Hill RD. Very mild senile dementia of the Alzheimer type. II. Psychometric test performance. Arch Neurol 1989;46:383-386.

20. Morris JC, McKeel DW Jr, Storandt M, et al. Very mild Alzheimer's disease: informant-based clinical, psychometric, and pathologic distinction from normal aging. Neurology 1991;41:469-478.
21. Koss E, Patterson MB, Ownby R, Stuckey JC, Whitehouse PJ. Memory evaluation in Alzheimer's disease. Caregivers' appraisals and objective testing. Arch Neurol 1993;50:92-97.

22. Crook T, Bartus RT, Ferris SH, Whitehouse P, Cohen GD, Gershon S. Age-associated memory impairment: proposed diagnostic criteria and measures of clinical change-report of a National Institute of Mental Health Work Group. Dev Neuropsychol 1986;2:261-276.

23. Koivisto K, Reinikainen KJ, Hänninen T, et al. The prevalence of age-associated memory impairment in a randomly selected population from eastern Finland. Neurology 1995; 45:741-747.

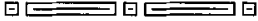

\title{
The P1 component of the middle latency auditory evoked potential predicts a practice effect during clinical trials in Alzheimer's disease
}

\author{
Joseph B. Green, MD; William W. Elder, BS; and David M. Freed, PhD
}

\begin{abstract}
Article abstract-Thirty-five patients with probable Alzheimer's disease who were enrolled in an experimental drug trial of linopirdine underwent repeated testing that included recording the middle latency auditory evoked potential (MLAEP), the Mini-Mental State Examination (MMSE), and the Alzheimer's Disease Assessment Scale Cognitive Subscale (ADASCOG). Patients lacking the P1 component of the MLAEP exhibited a significantly greater decline in cognitive function as measured by the ADASCOG over 56 weeks. This decline appeared to be due to a less robust practice effect, which was maximal in all patients at 16 weeks. At the end of 56 weeks the entire group of patients was near baseline with respect to the ADASCOG. This lack of the annualized decline expected from other longitudinal studies may be explained by practice and placebo effects. The MMSE did not exhibit a practice effect and showed the expected decline in scores.
\end{abstract}

NEUROLOGY 1995;45:962-966

Buchwald et al ${ }^{1}$ first reported the $\mathrm{P} 1$ component of the middle latency auditory evoked potential (MLAEP) to be absent in Alzheimer's disease (AD), and Green et $\mathrm{al}^{2}$ later confirmed this finding. We have found $\mathrm{P} 1$ to be absent in $47.5 \%$ of 101 patients with probable $\mathrm{AD}$ (unpublished data). The goal of the current study was to determine if $\mathrm{AD}$ patients lacking the $\mathrm{P} 1$ response differ with respect to clinical course and cognitive decline. In the course of this study, which lasted 56 weeks, we obtained important data concerning practice and placebo effects that may provide guidance in planning future clinical drug trials in AD.

Methods. The subjects of this investigation were 35 patients with probable $\mathrm{AD}$ who were enrolled in a clinical trial of a putative cognition-enhancing drug, linopirdine. Patients were receiving either drug or placebo and were followed for a total of 56 weeks. Patients were thoroughly evaluated with repeated neurologic examinations and imaging studies. The protocol of the study required the following to be completed at screening and during regular intervals: the Hachinski Ischemia Scale, the Mini-Mental State Examination (MMSE), the Hamilton Depression Scale, the Alzheimer's Disease Assessment Scale (ADAS), Word Recall task, Word Recognition task, Syndrom Kurztest, Instrumental Activities of Daily Living Scale (Lawton-Brody), Dementia Behavior Disturbance Scale, and Clinical Global Impressions. In addition, most patients also had neuropsychological testing prior to screening, commonly the CERAD battery. The diagnosis of probable $A D$ was made in accordance with NINCDS-ADRDA guidelines. ${ }^{3}$ All patients and guardians gave informed con-

From Texas Tech University Health Sciences Center, Department of Neurology, Lubbock, TX.

Supported by the Alzheimer's Disease Institute and the Tarbox Parkinson's Disease Institute.

Presented in part at the annual meeting of the American Electroencephalographic Society, Chicago, IL, September 1994.

Received June 3, 1994. Accepted in final form October 6, 1994.

Address correspondence and reprint requests to Dr. Joseph B. Green, Department of Neurology, Loyola Stritch School of Medicine, Hines VA Hospital, Building 1, Room 1A202B, P.O. Box 20, Hines, IL 60141. 